\title{
Wavelet-based Image Fusion for Enhancement of ROI in CT Image
}

\author{
Nguyen Thanh Hai \\ Faculty of Electrical-Electronic Engineering, UTE-HCMC, Vietnam; \\ nthai@hcmute.edu.vn
}

\begin{abstract}
This paper proposes a novel method for enhancing Computed Tomography (CT) image. In this proposed method, the entropy-based histogram algorithm was applied to detach significant values in CT image to produce a detached image. In addition, wavelet transforms were employed for both the original CT image and the detached image to create image fusion. Therefore, an enhanced image with tumor region was obtained for diagnosis using an inverse wavelet transform. The result is that the processed region in the enhanced image shows more prominent points than that in the original image. This result can allow physicians or doctors diagnose and predict more accurately tumor regions in the enhanced CT image.
\end{abstract}

Keywords: CT image, Wavelet Transform, Image Enhancement, Fusion Image.

\section{INTRODUCTION}

Cancer is a century disease which can occur in anyone's body. The most importance of cancer problem is to diagnose and detect it earlier for treatment. In practice, there are a few technologies of medical imaging such as Positron Emission Tomography (PET), Magnetic Resonance imaging (MRI), Computed Tomography (CT) and Ultrasound [1-3]. However, image processing is always applied for improving medical images. This allows doctors more easily diagnose and predict cancer diseases.

In biomedical image processing, different approaches have been proposed for detection of Regions of Interest (ROI) [4]. In lung image, segmentation algorithm was applied to enhance local anatomical structure using geometric and intensity models. In particular, morphologybased ROI segmentation was combined with watershed transform for comparative analysis in noisy environment [5]. A. Kumar, et al. developed a graph-based Content-based image retrieval (CBIR) method to exploit 3D spatial features extracted from volumetric ROIs [6]. In addition, 
authors applied a graph edit distance for measurement of the similarity of PET-CT images based on the spatial arrangement of tumors and organs.

Wavelet transform [7] has been applied for image processing to filter or to find features. An efficient algorithm for removing noise from corrupted images incorporating a wavelet-based threshold with a spatial based joint bilateral filter [8]. In this method, the performances for image denoising on a number of standard images were evaluated based on signal to noise ratio. Another application is that the author et al. applied wavelet transform with Haar function for image denoising [9]. The result is that image noise was effectively reduced and improved through the evaluation of the peak signal-to-noise ratio.

For Enhancement of medical image, a proposed method of pre-restoration adjustment phase was applied [10]. To low contrast images, an adaptive histogram equalization was employed with six stages. Moreover, authors tested this algorithm with over fifty medical images and also compared the processed image results with previous works. Finally, the conducted results with various features to illustrate the effectiveness of the proposed method. Another research is that a wavelet transform was employed for enhancement of CT image [11]. Authors tested the proposed method to 118 patients with cancers and 60 with benign tumors in this study. The results indicated that the image enhancement using the proposed method improved the diagnosis accuracy.

In this paper, for image enhancement to be better quality than an original CT image, histogram and entropy values of the image are processed and computed. Thus, in the proposed method, the remarkable region in the original image detached and then transformed using a wavelet transform with Harr function. In addition, the wavelet transform are employed to compute the original image to produce a wavelet image. A combined algorithm is applied to produce image fusion. An enhanced image is created after an inverse wavelet transform.

\section{METHOD}

In general, an original CT image with tumor region will be enhanced for diagnosis. Firstly, the original CT image is transformed to produce a histogram image. Based on this histogram image, one can calculate entropy statistics to obtain an image with significant values, called the detach image. This detach image and the original image are transformed to create image fusion using decomposition wavelet transforms. For diagnosis, the inverse wavelet transform is utilized to obtain an enhanced image as shown in Figure 1. 


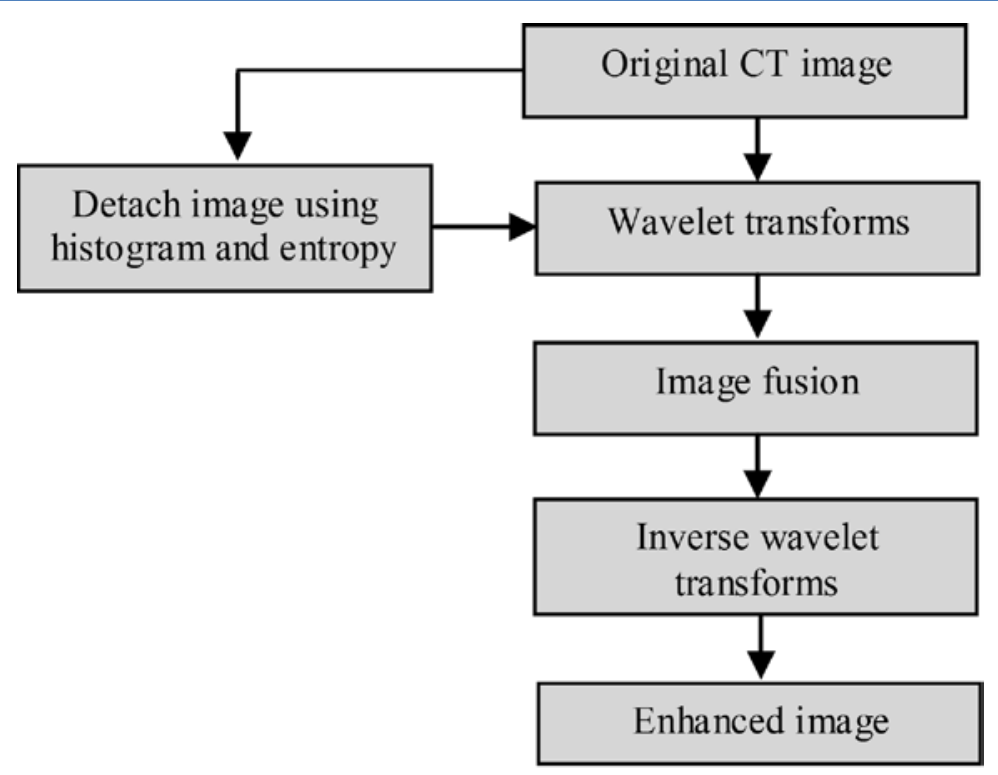

Figure 1: Block diagram of computing an enhancement image

The proposed method will be applied to process an original image of a patient with the circled region, in which there is Region of Interest (ROI), called the interrogative region to tumors, as shown in Figure 2. This region represents many details, in which there are some points addressed by arrows for enhancement.

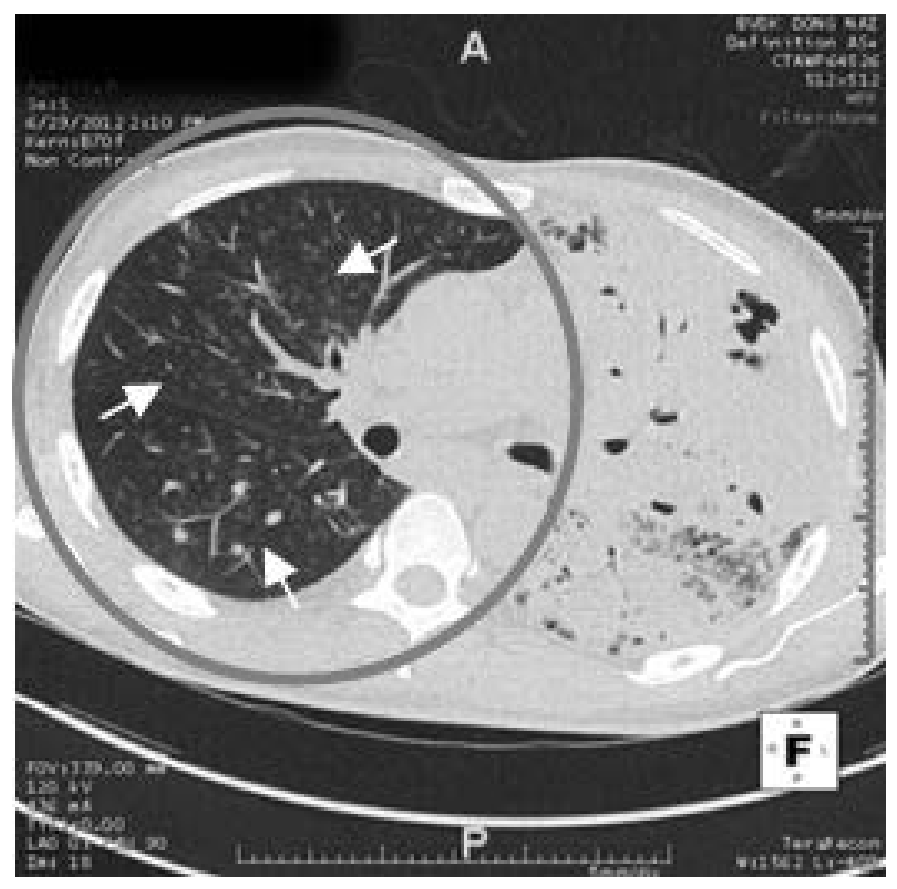

Figure 2: CT image of a patient (provided by Dong Nai hospital, Vietnam) 


\subsection{Detach of Image using Histogram and Entropy}

For enhancement of image using information of histogram and entropy, one needs to determine a histogram map. Consider an CT image $f(m, n)$ with the MxN size, in which gray levels $l=0,1,2, \ldots, 255$. The histogram of the image is defined as follows:

$$
\text { hist }_{f(m, n)}(l)=\sum_{m=0}^{M-1} \sum_{n=0}^{N-1} \delta_{d}(f(m, n)-l)
$$

where the discrete unit impulse function is expressed as the following function:

$$
\delta_{d}(k)=\left\{\begin{array}{cc}
1 & k=0 \\
0 & \text { otherwise }
\end{array}\right.
$$

In order to determine gray levels in an image, a histogram map is calculated as shown in Figure za using Eq. (1). The histogram may be normalized by dividing its entries by the total number of pixels in the image for image fusion. The normalized histogram, which may be taken to represent the probability density function $p_{f}(l)$ for generating the fused image, is expressed as follows:

$$
p_{f}(l)=\frac{1}{\sum_{l=0}^{255} h_{i s t} t_{f(m, n)}(l)} * \text { hist }_{f(m, n)}(l)
$$

where $p_{f}(l)$ denotes the normalized histogram, $f(m, n)$ represents the gray levels of the image, $l=0,1,2, \ldots, 255$ and $\sum_{l=0}^{255} p_{f}(l)=1$.
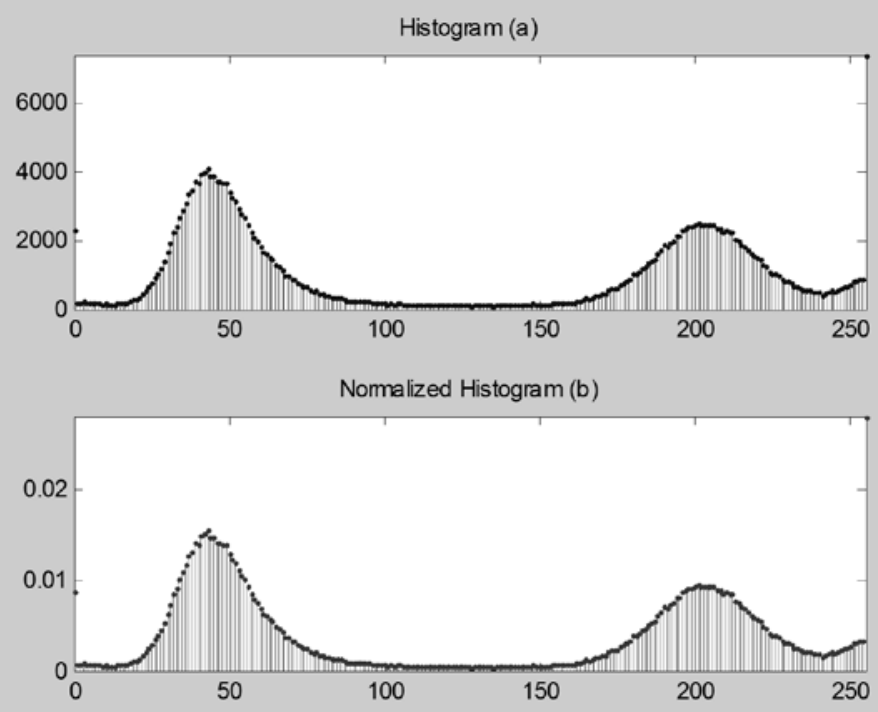

Figure 3: Representation of histogram images, (a) histogram of the original CT image with 256 gray-levels and pixels; (b) histogram after normalizing with 256 gray-levels and levels in [0 1]. 
Nguyen Thanh Hai; Wavelet-based Image Fusion for Enhancement of ROI in CT Image, Journal of Biomedical Engineering and Medical Imaging, Volume 1, Issue 4 Aug (2014), PP 1-13

Figure 3 shows two histogram maps with gray levels vs. pixel density, in which Figure $4 a$ is the histogram of the original image and Figure $3 b$ represents the histogram of the normalized image with the gray levels in [0 1] for determination of image information.

For calculation of image information $h\left(p_{1}\right), l=0,1,2, \ldots, 255$, one can measure conveyable information corresponding to each different gray level in the $2 \mathrm{D}$ image. This relates to the probability of gray levels in an image. Indeed, a totally unexpected event conveys maximal information when it occurs, where $p_{f}(l)=0$ for $h\left(p_{l}\right)=\infty$ and an event, that is certain to occur, does not convey any information, where $p_{f}(l)=1$ for $h\left(p_{l}\right)=0$. Then if the more $h\left(p_{l}\right)$ is small, the more gray-level will carry important transmitter information in image. Therefore, the set of the information values $h\left(p_{l}\right)$ can be determined using the following expression:

$$
h\left(p_{l}\right)=-\log \left(p_{f}(l)\right)
$$

Based on the probability of gray levels, its entropy $H$ is calculated for determining pixels with the highest information. The equation to calculate the entropy values is described as follows:

$$
H=-\sum_{l=0}^{255} p_{f}(l) * \log _{2}\left(p_{f}(l)\right)
$$

From information of the normalized histogram map and the entropy, one can determine values with the highest information of the image. Based on these information, the neighborhood pixels around these pixel values can be split to produce a detached image.

In particular, based on the normalized histogram, the gray values of the lowest probability can be determined. For example, assume that the gray level is 128 and the value of information $h(p)=0.004$ for $l=128$, it can be recognized that this is the important value in image and it should be detached for image enhancement. However, the problem is that how many points in the detached image is reasonable. In this case, one can use a coefficient, called $\Delta$ cof. If the selection of the coefficient $\Delta$ cof is appropriate, the processed image has good quality. Assume that $h\left(p_{1}\right)=0.004$ is chosen, it is very hard to distinguish the processed image and the original image due to differences between two entropies such as $H_{\Delta c o f}=7.2695$ (the processed image) and $H_{0}=7.2696$ (the original image). In this case, its error must be less than $2 \%$ (Weber's Law states that the ratio of the increment threshold to the background intensity is a constant). Therefore, we need to adjust this error by selecting the coefficient $\Delta$ cof to obtain a desired value for creating the high quality information in the image. Thereby, one can detach many important values in the image and the value of the information $h_{o}$ to be extracted is defined as follows:

$$
h_{o}=\min \left(h\left(p_{l}\right)\right)+\Delta c o f
$$


where $\Delta c o f=0.001, h_{o}=0.005$ is obtained. Therefore, we need to detach the information to be $h_{p(l)} \leq h_{o}$. From these values, a set of $Q(q)$ is obtained, where $q$ is the set of quantity $h_{p(l)}$, is satisfied the above conditions.

Table 1: Parameters of entropy information in image with $\Delta$ cof

\begin{tabular}{|c||c|c|c|c|c||}
\hline$\Delta$ cof & ho & $\mathrm{Q}(\mathrm{q})$ & $h_{p /}(\mathrm{min})$ & $h_{p /}(\mathrm{max})$ & $H_{\Delta c o f}$ \\
\hline \hline $\mathbf{0 . 0 0 0}$ & 0.004 & 1 & 128 & 128 & 7.2695 \\
\hline $\mathbf{0 . 0 0 1}$ & 0.005 & 11 & 115 & 142 & 7.2555 \\
\hline $\mathbf{0 . 0 0 2}$ & 0.006 & 45 & 107 & 149 & 7.1763 \\
\hline $\mathbf{0 . 0 0 3}$ & 0.007 & 62 & 101 & 155 & 7.1386 \\
\hline $\mathbf{0 . 0 0 4}$ & 0.008 & 76 & 96 & 161 & 7.0985 \\
\hline $\mathbf{0 . 0 0 5}$ & 0.009 & 86 & 11 & 161 & 7.0772 \\
\hline $\mathbf{0 . 0 2 0}$ & 0.024 & 147 & 3 & 250 & 6.7145 \\
\hline \hline
\end{tabular}

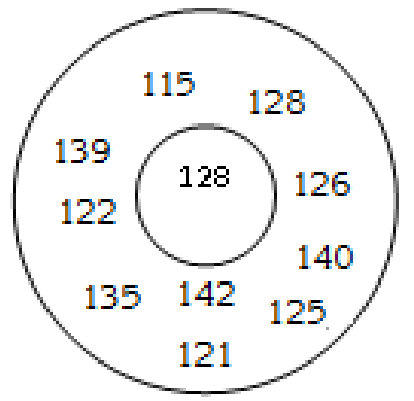

Figure 4: Neighborhood region the gray-level-value I= 128 which convey the highest information

When $Q(q)$ includes 11 different gray-level values, that are 115, 121, 122, 125, 126, 128, 135, 139,140 and 142 . These gray-level values are nearby with gray-level value $l=128$ and $h(p)=0.005$ that is presented in Figure 4. From these points, one will detach them from an original image in order to get a proposed detached image.

Table 2 shows a few cases with many different coefficients $\Delta$ cof selected for comparison and evaluation. When the coefficient $\Delta c o f$ is selected increasingly, the values of information $h\left(p_{1}\right)$ will increase. This means that the information of gray levels in the image are declined due to many sets of quantity in $Q(q)$ have no necessary information. Figures 5 a and 5 b represent detached images corresponding to different coefficients $\Delta$ cof and the object edges in Figure $5 \mathrm{~b}$ are better that in Figure 5 a. 


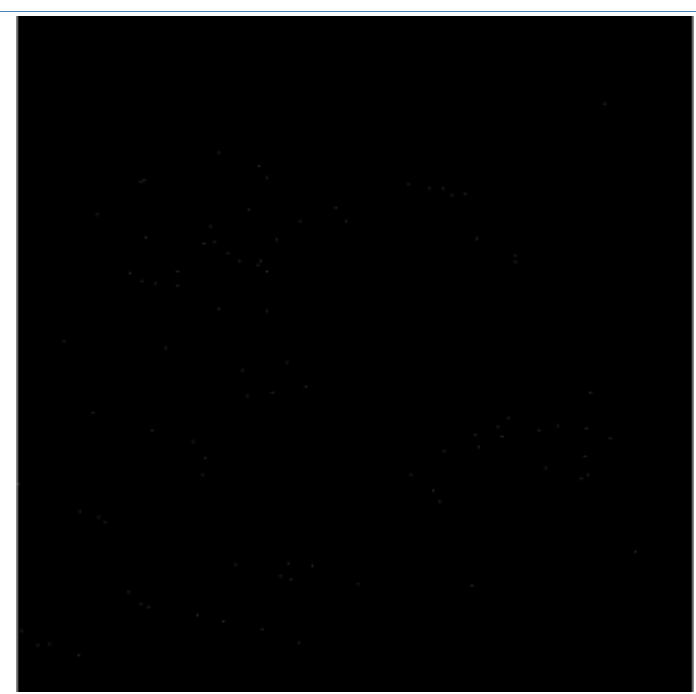

(a)

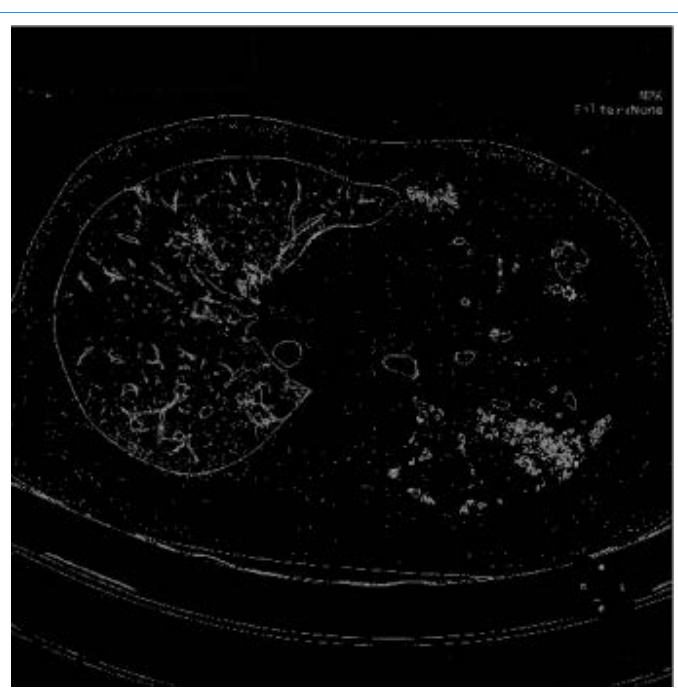

(b)

Figure 5: Detach image with different coefficients: (a) $\Delta c o f=0.000$; (b) $\Delta c o f=0.005$

From the histogram algorithm, the novel detach image algorithm, which is built, can detach each gray-level of the image to create the set of different gray-levels from o to 255 . Assume that $G$ is the set of 256 pixel values from 256 gray-levels in CT image and is expressed as follows:

$$
G=\sum_{m=0}^{M-1} \sum_{n=0}^{N-1} g_{l}(m, n)=\left(\delta_{d}(f(m, n)-l)\right) * l
$$

where $l=0,1,2, \ldots, 255$ respectively and $\delta_{d}$ is the discrete unit impulse function). From Eq. (7) we will get 256 arrays, every array includes one gray-level value and its position in image from o to 255. Assume that detach image based on $\Delta c o f f d(m, n)$ can be calculated by combining between the $\mathrm{G}$ matrix of gray levels and the values $Q(q)$ using the following formulas:

$$
\begin{gathered}
g_{L}(m, n)=g_{l}(m, n) *\left(\delta_{d}(l-Q(q))\right) \\
f_{d}(m, n)=\sum_{i=0}^{255} g_{l}(m, n)
\end{gathered}
$$

where $l=0: 255$ and $q=1$ :length[Q $(q)]$.

\subsection{Wavelet transforms with Harr function}

For image enhancement, wavelet transform with Harr function are utilized for both the detached image and the original image to produce wavelet images. Therefore, one detaches these two wavelet images to create image fusion. The purpose of Haar-type Wavelet Transforms (HWT) is to filter noise around the suspected areas. A CT image $f(m, n)$ can be considered as a matrix of the $M \times N$ image, in which columns $A$ denotes $a^{1}, a^{2}, \ldots, a^{N}$. The HWT applied for each column of $A$ is $C$, which is defined as follows: 


$$
C=W_{N} A=\left(W_{N} \mathrm{a}^{1}, W_{N} \mathrm{a}^{2}, \ldots, W_{N} \mathrm{a}^{N}\right)
$$

Therefore, the rows of $C$ are continuously processed and then multiply the second side of Eq. (11) and the matrix $W_{M}^{T}$, one has:

$$
C W_{M}^{T}=W_{N} A W_{M}^{T}
$$

Thus, the 2D HWT of the $M \times N$ matrix $\mathrm{A}$ is represented as follows:

$$
B=W_{N} A W_{M}^{T}
$$

where $B=C W_{M}^{T}$. Assume that $C T$ image is $f(m, n)$ and considered as a matrix of the $512 \times 512$ image. Thus, we can define $W_{512}=[\mathrm{H} \mid \mathrm{G}]$ and transform it to obtain as follows:

$$
W_{512} A W_{512}^{T}=\left[\frac{H}{G}\right] A\left[H^{T} \mid G^{T}\right]=\left[\begin{array}{cc}
H A H^{T} & H A G^{T} \\
G A H^{T} & G A G^{T}
\end{array}\right]
$$

where $H$ and $G$ are the $256 \times 512$ matrices.

$$
\begin{gathered}
H=\left[\begin{array}{cccccccc}
\frac{1}{2} & \frac{1}{2} & 0 & 0 & \ldots & \ldots & 0 & 0 \\
0 & 0 & \frac{1}{2} & \frac{1}{2} & \ldots & \ldots & 0 & 0 \\
\ldots & \ldots & \ldots & \ldots & \ldots & \ldots & \ldots & \ldots \\
0 & 0 & 0 & 0 & \ldots & \ldots & \frac{1}{2} & \frac{1}{2}
\end{array}\right] \\
G=\left[\begin{array}{cccccccc}
-\frac{1}{2} & -\frac{1}{2} & 0 & 0 & \ldots & \ldots & 0 & 0 \\
0 & 0 & -\frac{1}{2} & -\frac{1}{2} & \ldots & \ldots & 0 & 0 \\
\ldots & \ldots & \ldots & \ldots & \ldots & \ldots & \ldots & \ldots \\
0 & 0 & 0 & 0 & \ldots & \ldots & -\frac{1}{2} & -\frac{1}{2}
\end{array}\right]
\end{gathered}
$$

From Eq. (14), one divided image into four parts of HWT image (called $B, V, H$ and $D$ ). In particular, $H A H^{T}(B)$ is an approximation of the original image; $G A G^{T}(H)$ can be viewed as differences along the rows of the image; similarly $H A G^{T}(\mathrm{~V})$ are differences along the columns of image and the fourth part is that $G A G^{T}(D)$ are described as differences along the diagonal of image. The objective of this method is to release the area $D$ due to it is the noisy area in this image.

Using two levels of the Wavelet transforms in Eq. (12) with coefficients $H, G$ in Eq. (13) and Eq. (14) for the original image $f(m, n)$ and the detached image $f_{d}(m, n)$. The result is that $f_{w}(m, n)$ and $f_{w d}(m, n)$ are obtained as shown in Fig. 6 and Fig.7. 


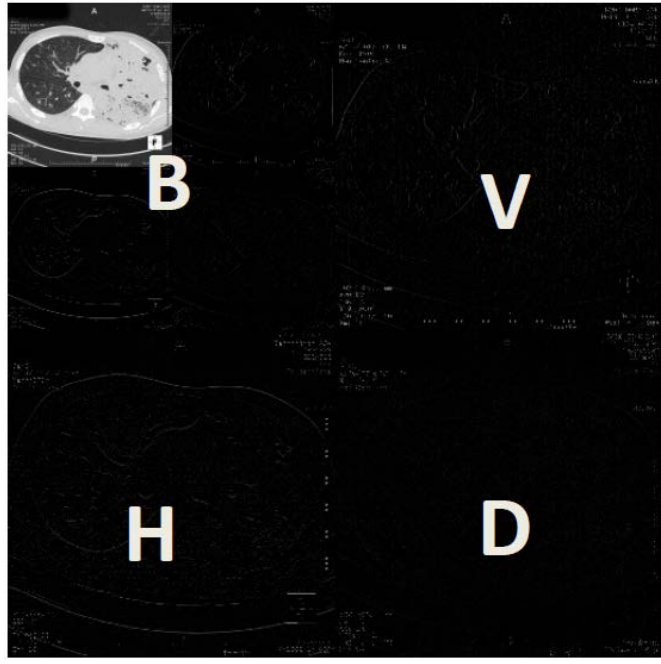

Figure 6: Original image with two HWT levels

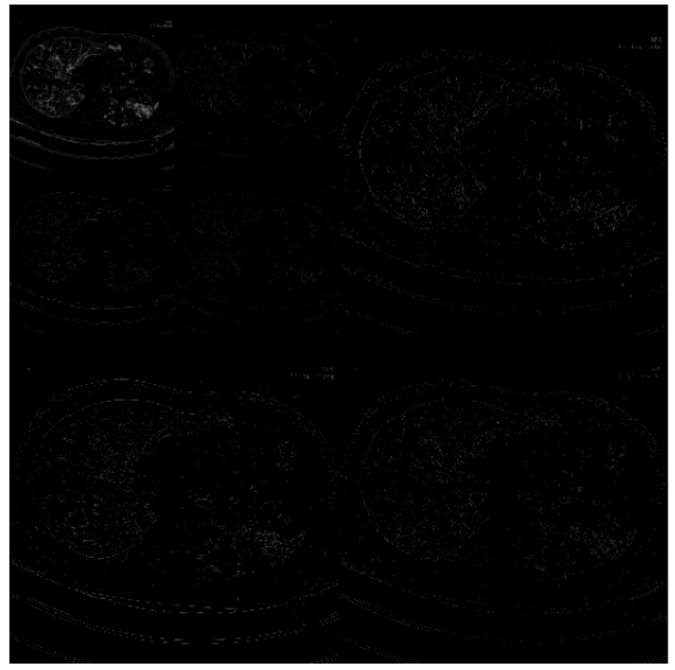

Figure 7: detached image with two HWT levels

\subsection{Image fusion}

In this paper, the wavelet transform method was applied to combine images to produce the fused image. The fusion process is that the input images are decomposed into the multiscale edge representation using the HWT in the different wavelet domains. In addition, the fused multi-scale representation is built based on pixel-by-pixel to produce the fused image and it is computed using the following expression:

$$
f_{\text {fusion }}(m, n)=\left\{\begin{array}{cc}
f_{w}(m, n)+f_{w d}(m, n) & f_{w}(m, n)+f_{w d}(m, n)<255 \\
255 & f_{w}(m, n)+f_{w d}(m, n) \geq 255
\end{array}\right.
$$

The advantage of this algorithm is easily performed. However, it would be difficult to observe them and the loss of gray-level values in image can occur if the gray-level values have identical positions. That will impact to other neighboring regions in image. But, we just concern with the marked locations, so it is temporarily ignored and the obtained image still give an effective image.

\subsection{Inverse wavelet transform algorithm}

In order to reconstruct the original image with enhancement for more easily observation, the inverse wavelet transforms is employed. From Eq. (11) and Eq. (12), an enhancement image is described using the following equation:

$$
f_{\text {final }}(m, n)=\left(W_{512}\right)^{\prime} f_{\text {fusion }}(m, n)\left(W_{512}{ }^{T}\right)^{\prime}
$$

where $W^{\prime}$ is the inverse matrix transform and $W^{T}$ is the transposition matrix.

\section{RESULTS AND DISCUSSION}

From the original CT image as shown in Fig. 8, the iterative procedure is performed in this method to process several lung cancer CT images with the different coefficients $\Delta$ cof . Moreover, this method, which depends on the entropy value of gray-levels in the image, is 
employed around the area which appears small details with blur related to the tumor problem. The result is that the enhanced images are represented corresponding to the coefficients $\Delta$ cof as shown in Figures 9 - 11. Each image with a different coefficient will give an enhanced image and it depends on doctors' choice for diagnosis.

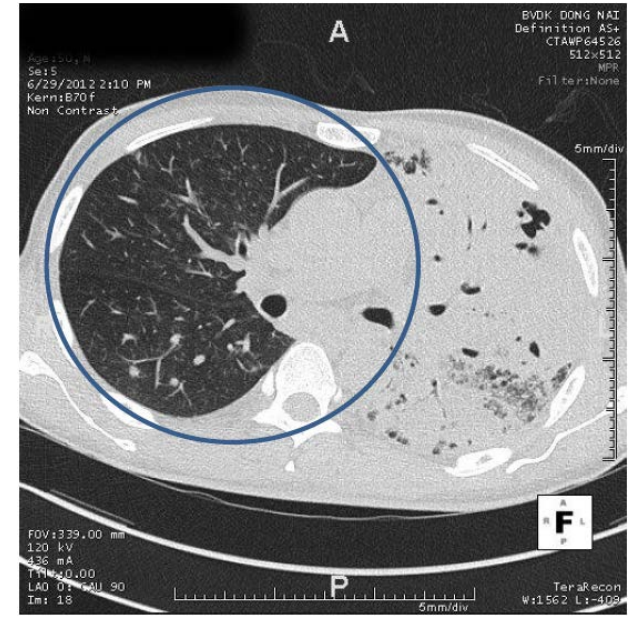

Figure 8: Original CT image of patient predicted lung cancer

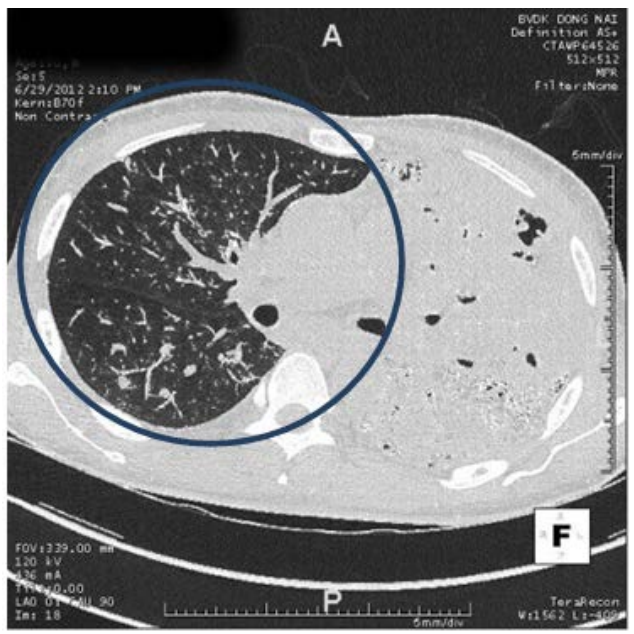

Figure 9: Enhanced CT image with coefficient $\Delta$ cof $=0.003$

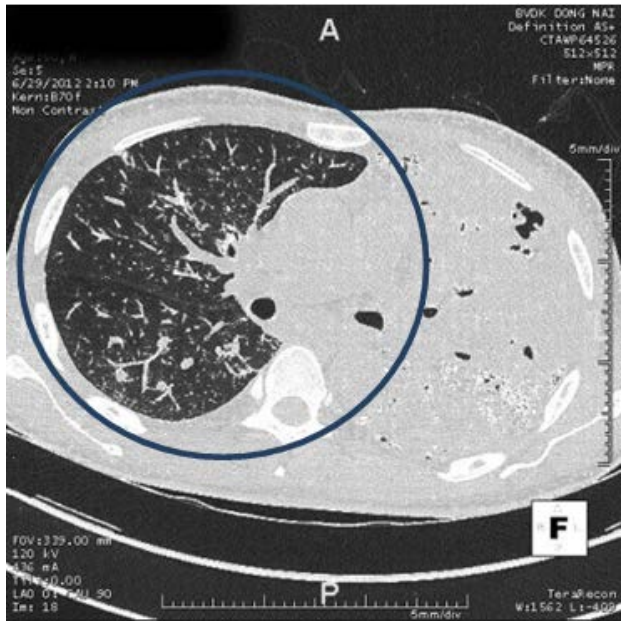

Figure 10: Enhanced image with coefficient $\Delta \operatorname{cof}=0.005$

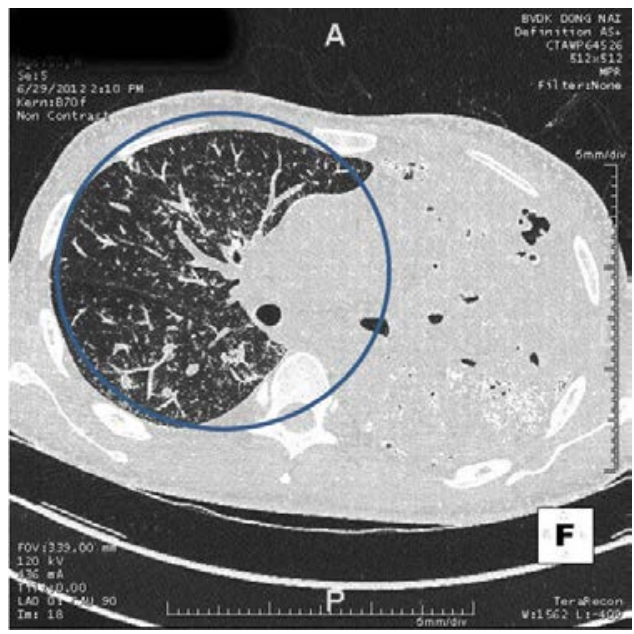

Figure 11: Enhanced CT image with coefficient $\Delta \operatorname{cof}=0.009$

In comparison between Figure 9, Figure 10 and Figure 10, the initial requirement is that the $\mathrm{ROI}$ with the enhanced details has been found to be sharper and clearer. Moreover, details of vascular, pulmonary capillaries in the lung have been clarified. Additions to other areas, the outside of the circle, which do not need to consider due to the objective of the paper just aims to process remarkable regions in $C T$ image. Since the process of biopsy, diagnosis will be followed by a team of researchers and doctors to make decision of the most accurate diagnosis for patients. In this research, the coefficient $\Delta$ cof plays an important role due to it decides the 
Nguyen Thanh Hai; Wavelet-based Image Fusion for Enhancement of ROI in CT Image, Journal of Biomedical Engineering and Medical Imaging, Volume 1, Issue 4 Aug (2014), PP 1-13

detached image with the set of gray level quantity $Q(q)$ related to the quality level of enhancement image.

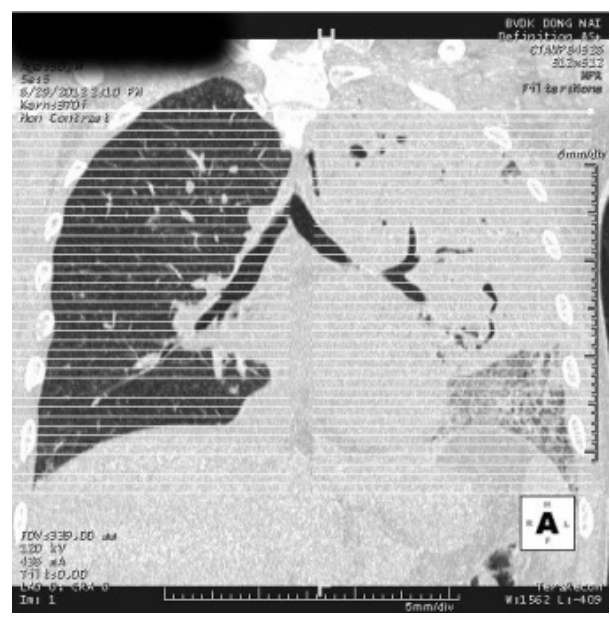

(a)

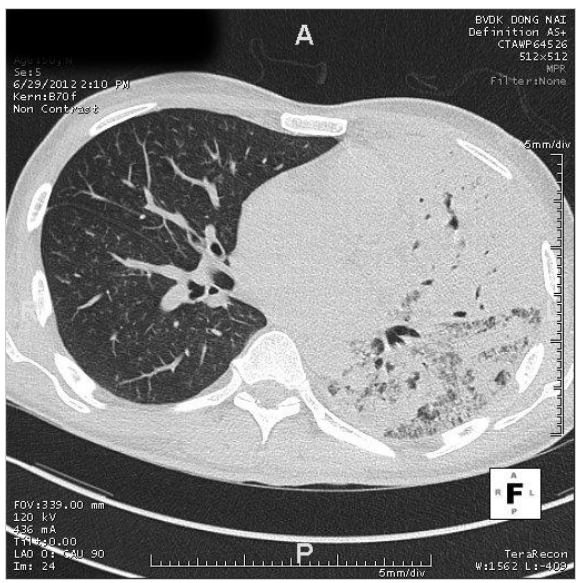

(c)

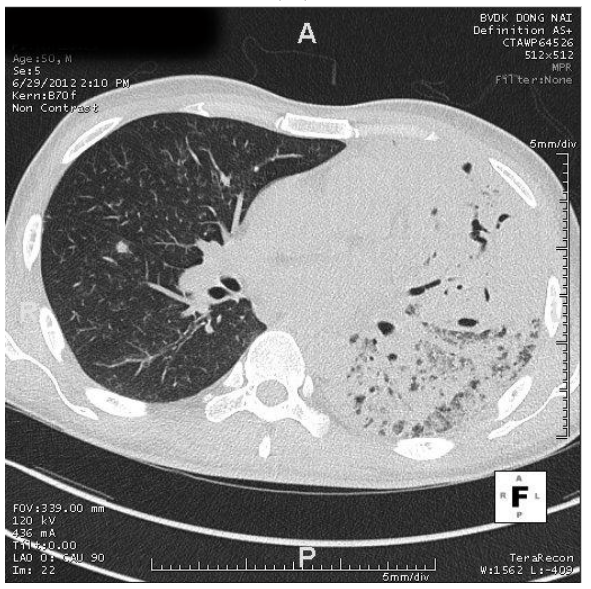

(e)

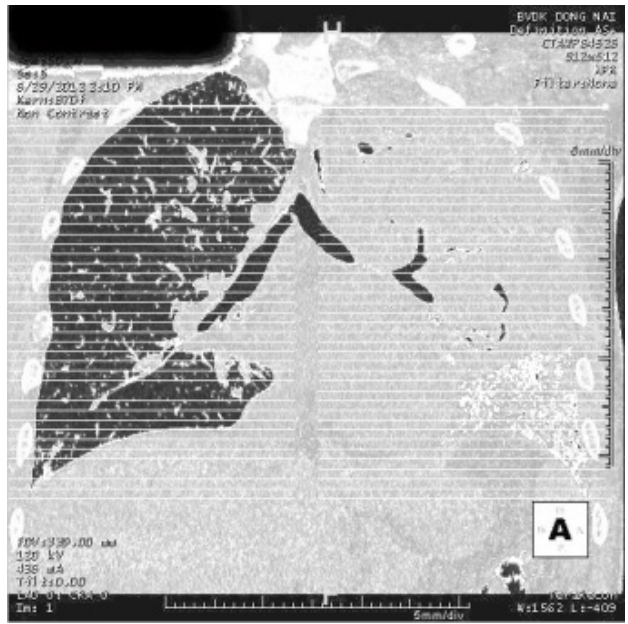

(b)

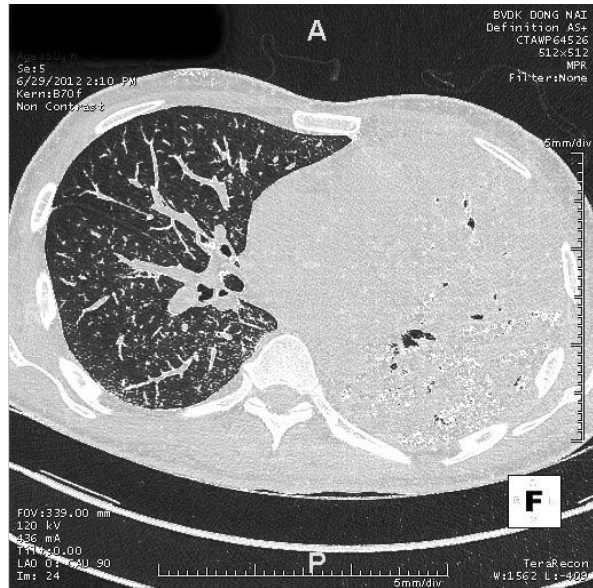

(d)

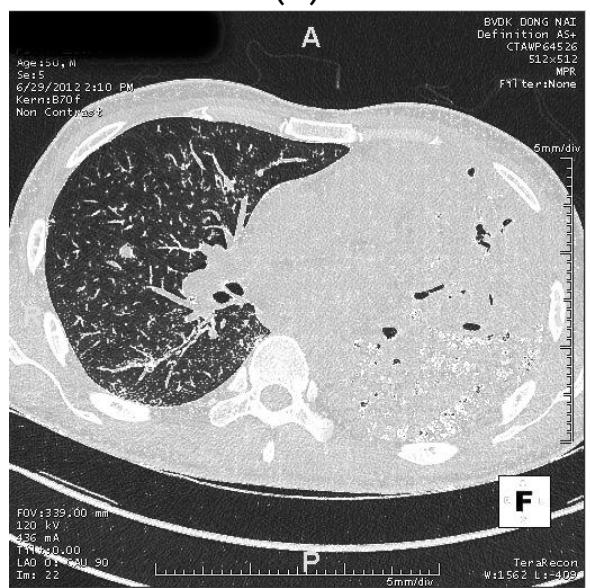

(f)

Figure 12: (a),(c) and (e) are the original CT images of a patient predicted lung cancer;

(b),(d) and (f) are the CT images processed with coefficient $\Delta \operatorname{cof}=0.007$ 
In order to increasingly evaluate the proposed method, more different results are shown in Figure 12, in which Figures (a), (c) and (e) are original images and Figures (b), (d) and (f) are images after processing with the coefficient $\Delta c o f=0.007$.

On the issue of relating to enhancement image, how to select the optimal coefficients $\Delta c o f$ and the most accuracy is very important. One found that the $(>0.02)$ ratio is too high or $(>0.005)$ is too, the image points, which are too bright, glitzy, high contrast, are so many unnecessary during image processing or they do not affect the enhanced image. Because these image points can be the lack of necessary information when the low coefficient values $\Delta$ cof are chosen.

\section{CONCLUSION}

This paper showed an image fusion novel method based on the Wavelet Transform with Harr function (HWT). In particular, the detached image developed from a histogram entropy approach was matched with an original image through the HWT to produce image fusion. In this image fusion method, several techniques were used such as optical expression, contrast coefficient, histogram, entropy, gray-scale transforms and gray-scale windows to create an enhanced image. The enhanced image showed brilliant points and regions of interest (ROIs) with good quality compared with the original image. Simulation results showed to illustrate the effectiveness of the proposed method.

\section{ACKNOWLEDGMENT}

We would like to thank Dong Nai hospital for providing these CT image. Furthermore, an honorable mention goes to a patient for his support on us in completing this project.

\section{REFERENCES}

[1]. J. E. Mackewn, G. Charles-Edwards, and J.J. Totman, P. Halsted, E.J. Somer, S.F. Keevil, P.K. Marsden "A fiducial marker based technique for alignment of simultaneously acquired PET and MRI images," in Nuclear Science Symposium Conference Record 2009.

[2]. E. A. Hoffman, J. M. Reinhardt, and M. Sonka, "Characterization of the interstitial lung diseases via densitybased and texture-based analysis of computed tomography images of lung structure and function," Academic Radiology, vol. 10, pp. 1104-1118, 2003.

[3]. L. J. Brattain, C. Floryan, O. P. Hauser, and M. Nguyen, "Simple and effective ultrasound needle guidance system," in The Annual International Conference of the IEEE on Engineering in Medicine and Biology Society, 2011. 
Nguyen Thanh Hai; Wavelet-based Image Fusion for Enhancement of ROI in CT Image, Journal of Biomedical Engineering and Medical Imaging, Volume 1, Issue 4 Aug (2014), PP 1-13

[4]. J. Huang, F. Jian, H. Wu, and H. Li, "An improved level set method for vertebra CT image segmentation," BioMedical Engineering OnLine, 2013.

[5]. V.V Kishore and R.V.S. Satyanarayana, "Performance evaluation of edge detectors - morphology based ROI segmentation and nodule detection from DICOM lung images in the noisy environment," in The 2013 IEEE 3rd international conference on Advance Computing Conference, 2013.

[6]. A. Kumar, J. Kim, L. Wen, D. Feng, "A Graph-based approach to the retrieval of volumetric PET-CT lung images," in The 2012 Annual International Conference of the IEEE on Engineering in Medicine and Biology Society (EMBC), 2012.

[7]. HaozhengRen, YihuaLan, and Y. Zhang, "Research of multi-focus image fusion based on M-band multiwavelet transformation," in The 2011 Fourth International Workshop on Advanced Computational Intelligence, 2011.

[8]. V.C. Bibina, V. S., and S. Viswasom,"Adaptive wavelet thresholding and joint bilateral filtering for image denoising," in The 2012 Annual IEEE India Conference (INDICON), 2012.

[9]. Y. Qiang, "Image denoisingbased on Haar wavelet transform," in The 2011 International Conference on Electronics and Optoelectronics (ICEOE), 2011.

[10]. Zohair Al-Ameen, Ghazali Sulong, Md. Gapar Md. Johar, "Employing a Suitable Contrast Enhancement Technique as a pre-restoration adjustment phase for Computed Tomography Medical Image", International Journal of Bio-Science and Bio-Technology, Vol. 5, No. 1, Feb, 2013.

[11]. Xiuhua G., Xiangye L., Huan W., Zhigang L., Wei W., Qian H., Kuncheng L., Wei W., "Enhanced CT Image by the Wavelet Transform Improving Diagnostic Accuracy of Chest Nodules", Journal of Digital Imaging, Vol. 24, No. 1, 2011. 